\title{
Using collocation and a hierarchical basis to solve the vibrational Schrödinger equation
}

\author{
Emil J. Zak and Tucker Carrington Jr. ${ }^{\text {a) } 1}$ \\ Chemistry Department, Queen's University, \\ Kingston, ONT, KrL 3N6, Canada
}

We show that it is possible to compute vibrational energy levels of polyatomic molecules with a collocation method and a basis of products of one-dimensional harmonic oscillator functions pruned so that it does not include functions for which the indices of many of the one-dimensional functions are non-zero. Functions with many non-zero indices are coupled only by terms that depend simultaneously on many coordinates and they are typically small. The collocation equation is derived without invoking differences of interpolation operators, which simplifies implementation of the method. This, however, requires inverting a matrix whose elements are values of the pruned basis functions at the collocation points. The collocation points are the points on a Smolyak grid whose size is equal to the size of the pruned basis set. The Smolyak grid is built from symmetrized Leja points. Because both the basis and the grid are not tensor products, the inverse is not straightforward. It can be done by using so-called hierarchical 1-D basis functions. They are defined so that the matrix whose elements are the 1-D hierarchical basis functions evaluated at points is lower triangular. We test the method by applying it to compute 100 energy levels of $\mathrm{CH}_{2} \mathrm{NH}$ with an iterative eigensolver.

a) emil.j.zak@gmail.com,tucker.carrington@queensu.ca 


\section{INTRODUCTION}

In recent years collocation has been used to compute vibrational spectra of small polyatomic molecules. ${ }^{1-9}$ To use collocation, one demands that a basis representation of solutions to the Schrödinger equation exactly satisfies the Schrödinger equation at a set of collocation points. In a collocation calculation, the potential matrix is diagonal, but the collocation eigenvalue problem is not the same as the eigenvalue problem solved when using a discrete variable representation (DVR). ${ }^{10,11}$ In a standard DVR calculation, the Hamiltonian matrix is symmetric and its rows and columns are labelled by DVR points. In a collocation calculation, the Hamiltonian matrix is non-symmetric and its rows are labelled by points and its columns by basis functions. Collocation does not require transforming vectors from a DVR to a finite basis representation (FBR) and then back from the FBR to the DVR. The standard collocation eigenvalue equation for a 1-D Schrödinger equation is (atomic units are used throughout the paper $)^{1}$,

$$
\left[-\frac{1}{2 m} \mathbf{B}^{\prime \prime}+\mathbf{V}^{\mathbf{d}} \mathbf{B}\right] \mathbf{U}=\mathbf{B U E}
$$

where $\mathbf{B}^{\prime \prime}$ is the matrix whose elements are second derivatives of basis functions evaluated at collocation points, $\mathbf{V}^{\mathbf{d}}$ is a diagonal matrix whose diagonal elements are values of the potential at the collocation points, and $\mathbf{B}$ is the matrix whose elements are the values of basis functions at collocation points. Everywhere in this paper, the number of points is equal to the number of basis functions. By left multiplying Eq. (1) by $\mathbf{B}^{\mathbf{T}} \mathbf{W}$, where $\mathbf{W}$ is a diagonal matrix of weights, one obtains an equation that looks deceptively like an FBR eigenvalue problem. They are different because in a standard FBR calculation ${ }^{11}$ only matrix elements of the potential are evaluated by quadrature; matrix elements of the kinetic energy operator (KEO) and the overlap matrix are exact. In this paper, we use Eq. (1) and no weights are necessary.

Collocation has important advantages. 1) There are no integrals and therefore no need for quadrature weights. It is not necessary to choose collocation points that could (with some weights) be used to accurately compute FBR potential and overlap matrix elements. When using Smolyak ideas for quadrature (not collocation), it is necessary to use enough

points to accurately evaluate overlap matrix elements. ${ }^{12-14}$ 2) Wavefunctions (and their corresponding energies) that can be exactly represented in the basis can be computed with any set of collocation points (however, some choices can lead to numerical problems). ${ }^{15} 3$ ) 
It is not necessary to calculate matrices that represent coordinate dependent coefficients of the KEO in the basis. For example, the KEO may be written so that it contains terms with second derivatives on the right and coordinate dependent coefficients $G_{c^{\prime}, c}$ on the left. For a vibrational problem, there may be as many as $\left(3 N_{a t}-6\right)^{2}$ such coefficients; $N_{a t}$ is the number of atoms. 4) There is no reason to choose coordinates and basis functions that make it possible to exactly compute matrix elements of terms or factors in the KEO. Nothing makes using non-orthogonal basis functions more difficult. Collocation has the disadvantage that the matrix for which one must compute eigenvalues is non-symmetric.

Collocation does nothing to mitigate the basis size problem; it is merely a means of obtaining energies and wavefunctions from a basis and a set of points. If the basis is huge, so also is the matrix. In FBR and DVR calculations, it is common to use a direct product basis. ${ }^{11,16-25}$. An important advantage of a direct product basis is that it allows one to efficiently evaluate the matrix-vector products (MVPs), required to use an iterative eigensolver. ${ }^{11,18,19,26,27}$ When both the grid and the basis are direct products, the direct product structure can be exploited by doing sums sequentially. A direct product basis also faciliates the matrix-vector products required in a collocation calculation. The problem with a direct product basis is, of course, its size which scales exponentially with $D$, the dimensionality of the problem. As a consequence, the memory required to store vectors, the cost of a single MVP, and the required number of MVPs all become large as $D$ increases. These problems are especially important for systems with more than 5 atoms.

In this paper, we use collocation and basis functions that are products of 1-dimensional (1-D) functions, but the basis is not a direct product basis. It is pruned, i.e. some of the direct product functions are removed. ${ }^{14,28-35}$ The advantage of the pruned basis is that it is much smaller. It is, however, non-trivial to use a pruned basis with collocation. One needs to identify a collocation grid that has the same number of points as the pruned basis has functions. It is also necessary to develop ideas for evaluating MVPs. If the basis and grid are not direct products then the indices one sums over to evaluate MVPs are interdependent and, unless one is careful, this jeopardizes sequential summation. ${ }^{3,14,32}$ In Ref. 3, a pruned collocation method is proposed and applied to compute vibrational energy levels of HONO. The collocation grid is a Smolyak grid. ${ }^{36-38}$ In Ref. 3 the theory is developed by starting with a Smolyak or sparse grid interpolant that is written as a sum of tensor products of differences between 1-D interpolation operators each of which is written in terms of Lagrange 
type functions. ${ }^{39}$

The method of Ref. 3 is efficient, because all sums are evaluated sequentially, but it has two deficiencies: 1) the equations are complicated; 2) the intermediate vectors generated by doing sums sequentially have more elements that there are basis functions and grid points, which significantly increases the memory cost of the calculation. In Ref. 4, the authors explain that by using the so-called "hierarchical" functions introduced in Ref. 3, it is possible to obviate the need for vectors with more elements than basis functions. These ideas were applied to compute vibrational energy levels of methane in Ref. 5 . In both Refs. 4 and 5, the formulae are derived from an interpolant made with Lagrange-type functions and written as a sum of tensor products of differences between 1-D interpolation operators. In this paper, we show that it is possible to use a much simpler approach. It also employs hierarchical functions, sequential summation and intermediate vectors with as many elements as there are basis functions, however, it does not explicitly use Lagrange-type functions and differences of interpolation operators. To work with a regular, rather than a generalized eiqenvalue problem, it is necessary to transform from a vector labelled by grid indices to a vector labelled by basis indices. A similar transformation ${ }^{40}$ has recently been used to implement a pruned multi-configuration time-dependent Hartee (MCTDH) method ${ }^{41}$. In the MCTDH calculation $^{40}$, one requires both the transformation from a basis vector to a grid vector and also the transformation from a grid vector to a basis vector. We demonstrate that the new procedure works by calculating the lowest 100 vibrational energy levels of methylenimine $\left(\mathrm{CH}_{2} \mathrm{NH}\right) . \mathrm{CH}_{2} \mathrm{NH}$ has been detected in the inter-stellar medium ${ }^{42}$ and is considered as a potential amino-acid formation substrate in space ${ }^{43,44}$.

\section{COLLOCATION EQUATIONS, COLLOCATION GRID, AND BASIS SET}

Regardless of whether or not the basis is a direct product and the collocation points are points on a direct product grid, when the kinetic energy operator can be written in the form $\hat{K}=\sum_{c=1}^{D} A_{c} \frac{\partial^{2}}{\partial{ }^{c} q^{2}}\left({ }^{c} q\right.$ is the $c$-th coordinate), the collocation equation can be written as

$$
\left(\mathbf{B}^{\prime \prime}+\mathbf{V}^{\operatorname{diag}} \mathbf{B}\right) \mathbf{U}=\mathbf{B U E} .
$$

or

$$
\mathbf{H Z}=\left(\mathbf{B}^{\prime \prime} \mathbf{B}^{-1}+\mathbf{V}^{\text {diag }}\right) \mathbf{Z}=\mathbf{Z E}
$$


if $\mathbf{B}^{-1}$ exists. When each basis function is a product of $1-\mathrm{D}$ factors, $\mathbf{B}$ is a matrix whose rows are labelled by collocation points $k_{1}, \ldots, k_{D}$, whose columns are labelled by basis functions $n_{1}, \ldots, n_{D}$, and whose elements are

$$
\underset{n_{1}, \ldots, n_{D}}{\mathbf{B}_{k_{1}, \ldots, k_{D}}}={ }^{(1)} \mathbf{B}_{k_{1}, n_{1}} \cdot \ldots \cdot{ }^{(D)} \mathbf{B}_{k_{D}, n_{D}}
$$

where ${ }^{(c)} \mathbf{B}_{k_{c}, n_{c}}=\varphi_{n_{c}}^{(c)}\left(q_{k_{c}}\right), \varphi_{n_{c}}^{(c)}$ is a 1-D basis function for coordinate ${ }^{c} q$, and $q_{k_{c}}$ is a collocation point for coordinate $c$. Note that B is not necessarily a Kronecker product. $\mathbf{B}^{\prime \prime}=\sum_{c}{ }^{\mathbf{c}} \mathbf{B}^{\prime \prime}$, in Eq. (3), is a sum of matrices, one for each coordinate and ${ }^{\mathbf{c}} \mathbf{B}^{\prime \prime}$ is the matrix whose elements are

$$
{ }^{c} \mathbf{B}_{k_{1}, \ldots, k_{D}}^{\prime \prime}=A_{c} \cdot{ }^{(1)} \mathbf{B}_{k_{1}, n_{1}} \cdot \ldots, n_{D}{ }^{(c)} \mathbf{B}_{k_{c}, n_{c}}^{\prime \prime} \cdot \ldots \cdot{ }^{(D)} \mathbf{B}_{k_{D}, n_{D}}
$$

where ${ }^{(c)} \mathbf{B}_{k_{c}, n_{c}}^{\prime \prime}=\left.\frac{d^{2}}{d q^{2}} \varphi_{n_{c}}^{(c)}(q)\right|_{q=q_{k_{c}}}$.

The collocation points we use are the points of a Smolyak grid. ${ }^{12,13,37,45,46}$ We expect them to be good collocation points because they are good quadrature points. ${ }^{15}$ To make a Smolyak grid, we choose a sequence of 1-D grids for each of the coordinates and a restriction on level indices. A Smolyak quadrature is usually written as a sum of contributing grids, each of which is a tensor product of 1-D grids that are members of the sequences. ${ }^{36,37,45}$ Each contributing grid is labelled by a level for each of the coordinates. In this paper, the contributing grids are those for which $\sum_{c}{ }^{(c)} G\left(\ell_{c}-1\right) \leq b$ and $m_{\ell}$, the number of points in the $\ell$ th level, equals $\ell^{12,47} \cdot{ }^{(c)} G(\cdot)$ is a non-decreasing function and $b$ is a truncation parameter. The best choice of ${ }^{(c)} G\left(n_{c}\right)$ depends on zeroth-order energy levels, the coupling, and also on the number of energy levels one wishes to compute. ${ }^{12,48,49}$ When the 1 -D grid sequences are nested, one can do MVPs by summing sequentially, without summing over contributing grids. ${ }^{14}$ A grid is nested when all points in the grid for level $\ell$ are also in the grid for level $\ell+1$. The points on the union (Smolyak) grid satisfy

$$
{ }^{(1)} G\left(k_{1}\right)+{ }^{(2)} G\left(k_{2}\right)+\ldots+{ }^{(D)} G\left(k_{D}\right) \leq b .
$$

The indices $k_{c}$ have the values, $k_{c}=0, \cdots, b$. Owing to the fact that $m_{\ell}=\ell$, the set of points with $\ell$ points includes all the points in the set with $\ell-1$ points and one new point. To make sequences by adding a single point, when $\ell$ is increased by one, we use sequences of symmetric Leja points. ${ }^{4,5,50}$ For every coordinate: the point in $\ell=1$ is $q=0$, the points in $\ell=2$ are $\left\{0, q_{1},\right\}$, the points in $\ell=3$ are $\left\{0, q_{1},-q_{1}\right\}$, the points in $\ell=4$ are $\left\{0, q_{1},-q_{1}, q_{2}\right\}$, 
the points in $\ell=5$ are $\left\{0, q_{1},-q_{1}, q_{2},-q_{2}\right\}$, etc. Errors are slightly larger when $m_{\ell}$ is even because in that case the grid is not symmetric. ${ }^{50}$ Non-symmetrized Leja points are discussed in Refs. 51-53. The basis we use corresponds to the collocation points of Eq. (6). It is $\left\{\varphi_{n_{1}} \varphi_{n_{2}} \ldots \varphi_{n_{D}}\right\}_{\left(n_{1}, \ldots, n_{D}\right) \in \mathcal{G}}$, with

$$
\mathcal{G}=\left\{\left.\left(n_{1}, \ldots, n_{D}\right)\right|^{(1)} G\left(n_{1}\right)+{ }^{(2)} G\left(n_{2}\right)+\ldots+{ }^{(D)} G\left(n_{D}\right) \leq b\right\} .
$$

Individual basis set indices $n_{c}$ range from 0 to ${ }^{(c)} G^{-1}(b)$, where it is assumed that ${ }^{(c)} G(0)=0$, for $c=1, \ldots, D$.

\section{HIERARCHICAL BASIS SET}

We shall compute eigenvalues of $\mathbf{H}$ in Eq. (3) using an iterative eigensolver. This requires evaluating MVPs. They are more efficient, if we use a so-called hierarchical basis. Mathematicians have long used basis functions that are piecewise linear and have the property that functions in level $\ell$ are equal to zero at all points in levels $1,2, \ldots, \ell-1$. They call these piecewise linear functions "hierarchical" ${ }^{36}$ In Ref. 3 (see also Ref. 54), a prescription is given for making basis functions that have the property that functions in level $\ell$ are equal to zero at all points in levels $1,2, \ldots, \ell-1$, but that are not piecewise linear and instead, level by level, span the same space as any chosen basis $\varphi_{n_{c}}^{(c)}\left({ }^{c} q\right)$. We call these functions hierarchical. They are important because piecewise linear functions are poor if one wishes to represent smooth vibrational wavefunctions.

Hierarchical basis functions are defined with the following equation:

$$
\tilde{\varphi}_{n}(q)=\sum_{m=0}^{n} \tilde{A}_{n m} \varphi_{m}(q),
$$

where $\varphi_{m}(q)$ is one of any set of 1D basis functions; in this paper they are 1-D harmonic oscillator eigenfunctions. The matrix elements $\tilde{A}_{n m}$ are determined by requiring that the hierarchical basis functions be zero at grid points $q_{k}$ with indices smaller than the index of the hierarchical basis function:

$$
\tilde{\varphi}_{n}\left(q_{k}\right)=0 \quad k=0,1, \ldots, n-1 .
$$

Imposing condition 9 yields a system of linear equations for $\tilde{A}_{n m}$. It is solved, row by row, by setting $\tilde{A}_{n n}=1$. The resulting $\tilde{\varphi}_{n}(q)$ are then normalized. Elements in the upper triangle 
of $\mathbf{A}$ are zero. Figure 1 displays a set of hierarchical basis functions constructed from 1D harmonic oscillator eigenfunctions and symmetric Leja points (discussed in section II).

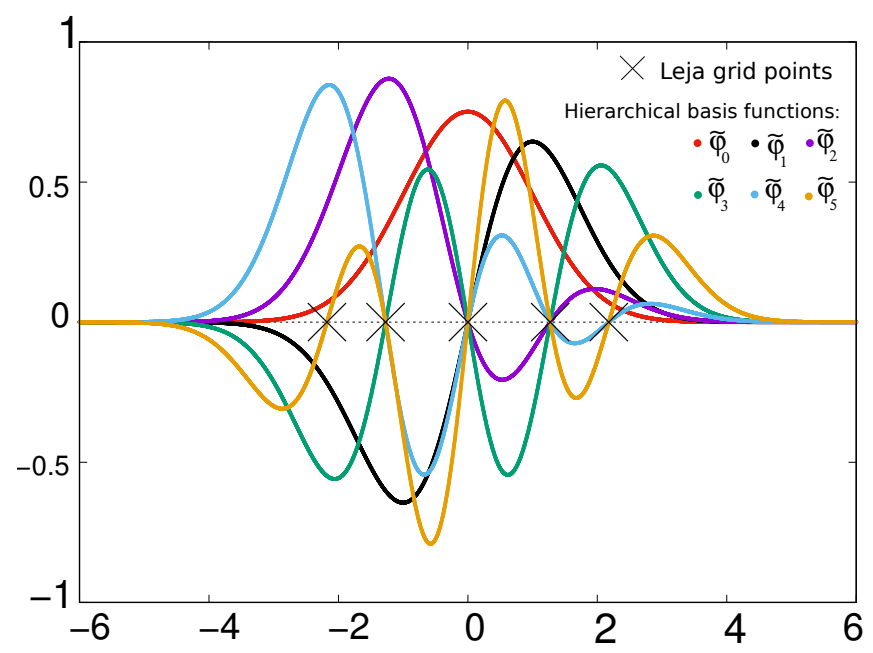

FIG. 1. Plot of the first six hierarchical basis functions $\tilde{\varphi}_{n}(q)$ generated from the 1D harmonic oscillator eigenfunctions. $\tilde{\varphi}_{n}(q)$ goes through $n$ Leja points (marked with crosses). The sixth Leja point is not shown because no function goes through it.

\section{COMPUTING MATRIX-VECTOR PRODUCTS WITH H}

$\mathbf{H}$ in Eq. (3) is not symmetric and we therefore use a re-started Arnoldi algorithm implemented in the ARPACK ${ }^{55}$ library to compute eigenvalues. To apply $\mathbf{H}$ to an Arnoldi vector $\mathbf{x}$, there are three steps: 1) $\left.{ }^{(1)} \mathbf{u}=\mathbf{V}^{\operatorname{diag}_{\mathbf{x}}} \mathbf{2} \quad 2\right)^{(2)} \mathbf{u}=\mathbf{B}^{-1} \mathbf{x}$; and $3{ }^{(3)} \mathbf{u}=\mathbf{B}^{\prime \prime}\left({ }^{(2)} \mathbf{u}\right)$. $\mathbf{H x}$ is the sum of ${ }^{(1)} \mathbf{u}$ and ${ }^{(3)} \mathbf{u}$. Because $\mathbf{V}^{\text {diag }}$ is diagonal, MVPs with it (step 1) are simple and cheap.

The MVP of step 2 is much more complicated. In Ref. 4, it is explained how to evaluate MVPs with $\tilde{\mathbf{T}}$ (see Ref. 4 for the definition of $\tilde{\mathbf{T}}$ ) using sequential summation and intermediate vectors of constant length. The equations exploit the lower triangularity of $\tilde{\mathbf{T}}$. In this paper, the collocation matrices ${ }^{(c)} \tilde{\mathbf{B}}_{k_{c} n_{c}}=\tilde{\varphi}_{n_{c}}^{(c)}\left(q_{k_{c}}\right)$ in the hierarchical basis are lower-triangular. Owing to their lower triangularity, we could use the method of Ref. 4 to do MVPs with $\tilde{\mathbf{B}}$. However, we need MVPs with $\tilde{\mathbf{B}}^{-1}$. Fortunately, similar ideas can be used because elements of $\tilde{\mathbf{B}}^{-1}$ can be written as products of elements of matrices ${ }^{\left({ }^{c}\right)} \tilde{\mathbf{B}}^{-1}$ that are themselves lower triangular. One way to show this is to prove that $\tilde{\mathbf{B}}^{-1}$ can be obtained by removing rows and columns from the inverse of the Kronecker product ${ }^{(1)} \tilde{\mathbf{B}} \otimes \ldots \otimes^{(D)} \tilde{\mathbf{B}} .^{40}$ 
Once it is established that elements of $\tilde{\mathbf{B}}^{-1}$ can be written as products of elements of ${ }^{(\mathbf{c})} \tilde{\mathbf{B}}^{-1}$, step 2 is implemented by doing sums sequentially,

$$
{ }^{(2)} \mathbf{u}_{n_{1}, n_{2}, \ldots, n_{D}}=\sum_{k_{1}=0}^{k_{1}^{\max }}{ }^{(1)} \tilde{\mathbf{B}}_{n_{1} k_{1}}^{-1} \sum_{k_{2}=0}^{k_{2}^{\max }}{ }^{(2)} \tilde{\mathbf{B}}_{n_{2} k_{2}}^{-1} \ldots \sum_{k_{D}=0}^{k_{D}^{\max }}{ }^{(D)} \tilde{\mathbf{B}}_{n_{D} k_{D}}^{-1} \mathbf{x}_{k_{1}, \ldots, k_{D}}
$$

with $k_{c}^{\max }=\min \left(n_{c},{ }^{(c)} G^{-1}\left[b-\sum_{c^{\prime}=1}^{c-1}{ }^{\left(c^{\prime}\right)} G\left(k_{c^{\prime}}\right)-\sum_{c^{\prime}=c+1}^{D}{ }^{\left(c^{\prime}\right)} G\left(n_{c^{\prime}}\right)\right]\right)$, where ${ }^{(c)} G^{-1}$ is the inverse function to ${ }^{(c)} G$. Because ${ }^{(c)} G$ is a monotonic function, its inverse always exists. Figure 2 shows that when $D=2$ and both ${ }^{(1)} \tilde{\mathbf{B}}^{-\mathbf{1}}$ and ${ }^{(2)} \tilde{\mathbf{B}}^{-\mathbf{1}}$ are lower triangular, the length of the intermediate vectors is preserved. 

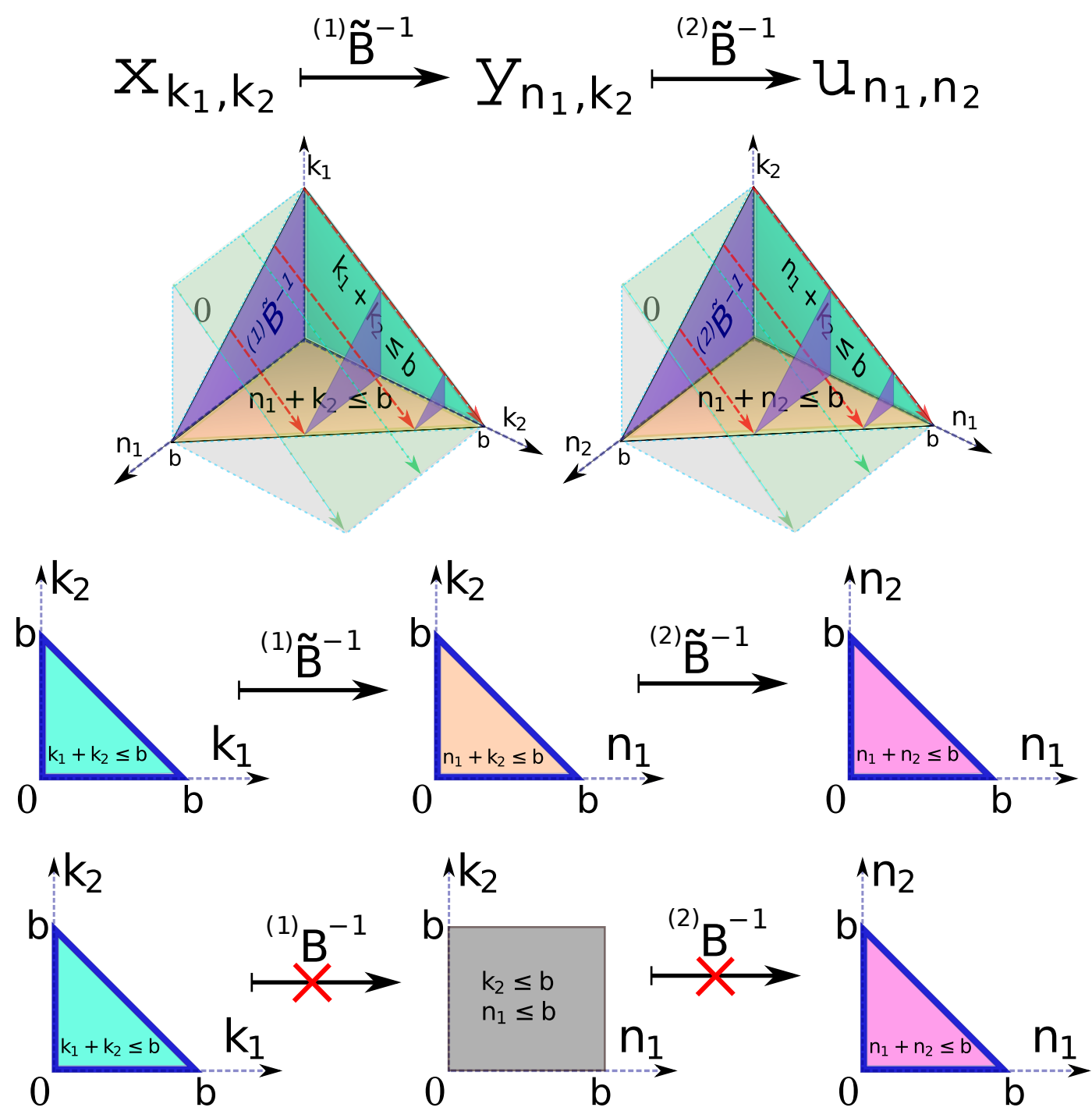

FIG. 2. Schematic representation of the grid $\rightarrow$ basis transformation $\tilde{\mathbf{B}}^{-\mathbf{1}}$ in two dimensions, with a basis set defined by the pruning condition $n_{1}+n_{2} \leq b$, and the corresponding grid indices satisfying $k_{1}+k_{2} \leq b$. The top panel depicts a chain of transformations as in eq.10. The middle panel shows that because ${ }^{(1)} \tilde{\mathbf{B}}^{-\mathbf{1}}$ and ${ }^{(2)} \tilde{\mathbf{B}}^{-\mathbf{1}}$ are lower triangular the indices of the intermediate vector (in the middle) are restricted by $n_{1}+k_{2} \leq b$. Once the index restrictions are applied, the matrices ${ }^{(1)} \mathbf{B}^{-\mathbf{1}}$ and ${ }^{(2)} \mathbf{B}^{-\mathbf{1}}$ (no tildes) are not square, and therefore their inverses do not exist. One might use pseudoinverses, but they would not be lower triangular and therefore the intermediate vector would be larger than the input and output vectors. This is indicated in the bottom panel.

MVPs with $\tilde{\mathbf{B}}^{\prime \prime}$ (step 3) can be done with similar ideas. Because the KEO we use is a simple sum of second derivatives, each term in $\tilde{\mathbf{B}}^{\prime \prime}$ has only one factor that is not lower 
triangular,

${ }^{(3)} \mathbf{u}_{k_{1}, k_{2}, \ldots, k_{D}}=\sum_{n_{1}=0}^{n_{1}^{\max }}{ }^{(1)} \tilde{\mathbf{B}}_{k_{1} n_{1}} \ldots \sum_{n_{c-1}=0}^{n_{c-1}^{\max }}{ }^{(c-1)} \tilde{\mathbf{B}}_{k_{c-1} n_{c-1}} \sum_{n_{c+1}=0}^{n_{c+1}^{\max }}{ }^{(c+1)} \tilde{\mathbf{B}}_{k_{c+1} n_{c+1}} \ldots \sum_{n_{D}=0}^{n_{D}^{\max }}{ }^{(D)} \tilde{\mathbf{B}}_{k_{D} n_{D}} \sum_{n_{c}=0}^{n_{c}^{\max }}{ }^{(c)} \tilde{\mathbf{B}}_{k_{c} n_{c}}^{\prime \prime}{ }^{(2)} \mathbf{u}_{n_{1}, n_{2}, \ldots, n_{D}}$.

The factor that is not lower triangular is ${ }^{(c)} \tilde{\mathbf{B}}^{\prime \prime}$. If the factor that is not lower triangular is put on the right, then MVPs can be efficiently evaluated by summing sequentially, without using intermediate vectors with more elements than there are basis functions. ${ }^{4}$ Upper limits on all the sums in eq. 11 except the sum over $n_{c}$, which is moved to the right, are

$$
n_{s \neq c}^{\max }=\min \left(k_{s},{ }^{(s)} G^{-1}\left[b-\sum_{c^{\prime}=1}^{s-1}{ }^{\left(c^{\prime}\right)} G\left(n_{c^{\prime}}\right)-\sum_{c^{\prime}=s+1}^{D}{ }^{\left(c^{\prime}\right)} G\left(k_{c^{\prime}}\right)\right]\right) .
$$

The upper limit for the sum over $n_{c}$ is

$$
n_{c}^{\max }={ }^{(c)} G^{-1}\left[b-\sum_{c^{\prime}=1}^{c-1}{ }^{\left(c^{\prime}\right)} G\left(n_{c^{\prime}}\right)-\sum_{c^{\prime}=c+1}^{D}{ }^{\left(c^{\prime}\right)} G\left(k_{c^{\prime}}\right)\right] .
$$

Fortran90 codes that implement the method of this paper, in 3-D and in 9-D, and documentation for them are available online at www.emilzak.com. They require a PES provided by the user. The KEO must be in the form given before Eq. (2), with frequencies provided

by the user. The user must specify the $1-\mathrm{D}$ basis size, as well as the ${ }^{(c)} G\left(n_{c}\right)$ pruning functions. In the eigensolver part of the input file, the user must specify the maximum number of Arnoldi iterations, the number of requested eigenvalues, a tolerance for convergence of the eigenvalues, as well as the number of basis vectors used in the restarted Arnoldi procedure. The codes, named $h$-collD, where $D$ is the number of dimensions, are compatible with Intel Fortran compilers and have been parallelized with Intel MKL OpenMP libraries. The only non-trivial MVP, the one for $\mathbf{B}^{\prime \prime} \mathbf{B}^{-1}$ (see Eq. (3)) is parallelized at two levels. First, it is parallelized over the $D$ independent terms in the KEO. For each term in eq. 11, one sequentially calculates matrix-vector products with matrices ${ }^{(c)} \tilde{\mathbf{B}}_{k_{c} n_{c}}$ or ${ }^{(c)} \tilde{\mathbf{B}}^{\prime \prime}$. When doing these 1-D matrix-vector products, we calculate components of the output vector in parallel; this is the second level of parallelization.

\section{A. Mapping of multidimensional vector indices}

To implement, for example, Eq. (11) we store vectors labelled by 9 indices in arrays with a single index by using a mapping. When evaluating the sums, the multi-indices 
$\left(n_{1}, n_{2}, n_{3}, n_{4}, n_{5}, n_{6}, n_{7}, n_{8}, n_{9}\right)$ are converted into a mapping index. ${ }^{13}$ The map $m: \mathbb{W}^{D} \ni$ $\left(n_{1}, n_{2}, \ldots, n_{D}\right) \longmapsto m\left(n_{1}, n_{2}, \ldots, n_{D}\right) \in \mathbb{N}$ over the basis indices is:

$$
m\left(n_{1}, n_{2}, \ldots, n_{D}\right)=\sum_{c=1}^{D}(c) L\left(n_{c}\right)
$$

with

$$
{ }^{(c)} L\left(n_{c}\right)=\sum_{i_{c}=0}^{n_{c}-1} \sum_{i_{c-1}=0}^{{ }^{c} n_{c-1}^{\max }} \cdots \sum_{i_{2}=0}^{{ }^{c} n_{2}^{\max }{ }^{c} \sum_{i_{1}=0}^{\max }}
$$

where ${ }^{c} n_{s}^{\max }={ }^{(c)} G^{-1}\left[b-\sum_{k=c+1}^{d}{ }^{(k)} G\left(n_{k}\right)-\sum_{k=s+1}^{c}{ }^{(k)} G\left(i_{k}\right)\right] .{ }^{(c)} L\left(n_{c}\right)$ is the number of basis functions for which $n_{c}$ is between 0 and $n_{c}-1$.

\section{CALCULATING ENERGY LEVELS OF $\mathrm{CH}_{2} \mathrm{NH}$}

To test the new method we calculated 100 lowest vibrational energy levels of $\mathrm{CH}_{2} \mathrm{NH}$, using a quartic force field $\mathrm{PES}^{56}$ and different basis sizes. Eigenfunctions of the 1-D harmonic oscillator are used as a 1-D basis functions:

$$
\varphi_{n_{c}}\left({ }^{c} q\right)=N_{n_{c}} H_{n_{c}}\left({ }^{c} q\right) e^{-\frac{c_{q}^{2}}{2}}
$$

where $H_{n_{c}}\left({ }^{c} q\right)$ is the $n_{c}$-th Hermite polynomial, $N_{n_{c}}$ is the normalisation constant and ${ }^{c} q$ is a dimensionless coordinate. The harmonic wavenumbers are: $\omega_{c}$ for $\mathrm{CH}_{2} \mathrm{NH}$ are 3491.5, 3198.3, 3086.8, 1685.1, 1510.1, 1397.2, 1089.4, 1186.4, and $1101.9 \mathrm{~cm}^{-1} .{ }^{57}$ The order of the dimensionless coordinates $\left\{{ }^{c} q\right\}_{c=1, \ldots 9}$ is the same as the order of the harmonic wavenumbers. From the primitive harmonic oscillator basis and the Leja points, a hierarchical basis $\left\{\tilde{\varphi}_{n_{c}}\right\}_{c=1, \ldots 9}$ is formed using eq. 8. The ${ }^{(c)} G\left(n_{c}\right)$ functions constraining the basis set indices are the same for all coordinates and the same as those in Ref. 4:

$$
\begin{array}{r}
G(0)=0, G(1)=13, G(2)=22, G(3)=31, G(4)=34, G(5)=42, \\
G(6)=50, G(7)=58, G(8)=66, G(9)=74, G(10)=82 \\
G(11)=90, G(12)=98, G(13)=106, G(14)=114, G(15)=122 \\
G(16)=130, G(17)=140, G(18)=148, G(19)=156, G(20)=164
\end{array}
$$

The ${ }^{(c)} G\left(n_{c}\right)$ are chosen for the calculation of 100 vibrational energy levels of $\mathrm{CH}_{2} \mathrm{NH}$. The choice is guided by the principle that the PES does not strongly couple many coordinates and 
that therefore basis functions with many non-zero indices $n_{1}, n_{2}, \ldots, n_{D}$ are less important than basis functions with only few non-zero indices, even when the sum of the indices is the same in both case. ${ }^{4,12}$. This explains the super-linear $(G(n)>n)$ behaviour of $G(n)$ at low values of $n$ and sub-linear $(G(n)<n)$ behaviour at high values of $n$. Potential-optimized 1-D basis functions ${ }^{58}$ are also available in the computer code at www.emilzak.com. Potentialoptimized basis functions are linear combinations of harmonic oscillator basis functions and determined by diagonalizing 1-D cut Hamiltonians obtained by fixing all coordinates except one to the equilibrium values. For $\mathrm{CH}_{2} \mathrm{NH}$, the harmonic oscillator eigenfunctions are excellent. We use the force constants of Ref. 57 as interpreted in Ref. 56

\section{A. Vibrational energy levels calculations}

Table I lists the lowest ten and the hightest seven of the lowest 100 vibrational energy levels of $\mathrm{CH}_{2} \mathrm{NH}$ calculated with the present method and different basis set sizes. Calculations were done with AMD Opteron(tm) Processors $6386 \mathrm{SE} 2.8 \mathrm{GHz}$ using 64 cores (threads). In the ARPACK eigensolver (DNDRV1) program, the maximum number of Arnoldi vectors used (NCV) was set to 300 and the relative tolerance for convergence of eigenvalues (tol) was $10^{-8}$.

TABLE I: Vibrational energy levels of $\mathrm{CH}_{2} \mathrm{NH}$ calculated with different basis set sizes $(b=90,105,115)$. All energy levels are in $\mathrm{cm}^{-1}$.

\begin{tabular}{|c|c|c|c|c|c|}
\hline $\mathrm{b}$ & 90 & 105 & \multicolumn{3}{|c|}{$115(105-90)(115-105)$} \\
\hline Basis size & 22375 & 73060 & 146398 & & \\
\hline \multicolumn{6}{|l|}{ State ID } \\
\hline 1 (ZPVE) & 8852.101 & 8852.074 & 8852.072 & -0.027 & -0.002 \\
\hline 2 & 9957.541 & 9957.649 & 9957.625 & 0.108 & -0.024 \\
\hline 3 & 9968.493 & 9968.499 & 9968.490 & 0.006 & -0.009 \\
\hline 4 & 10053.407 & 10053.442 & 10053.419 & 0.035 & -0.023 \\
\hline 5 & 10249.716 & 10249.705 & 10249.691 & -0.011 & -0.014 \\
\hline 6 & 10347.101 & 10346.765 & 10346.765 & -0.336 & 0.000 \\
\hline 7 & 10521.918 & 10522.052 & 10522.040 & 0.134 & -0.012 \\
\hline 8 & 11074.916 & 11074.252 & 11074.466 & -0.664 & 0.214 \\
\hline
\end{tabular}




\begin{tabular}{lrrr}
9 & 11077.73711077 .20411077 .208 & -0.533 & 0.004 \\
10 & 11099.94611099 .37611099 .344 & -0.570 & -0.032 \\
$\vdots$ & & & \\
94 & 13357.42613351 .58713350 .741 & -5.839 & -0.846 \\
95 & 13373.19613355 .89613355 .983 & -17.300 & 0.087 \\
96 & 13384.88513382 .05913381 .944 & -2.826 & -0.115 \\
97 & 13401.93613400 .14613399 .963 & -1.790 & -0.183 \\
98 & 13405.91013401 .13513400 .845 & -4.775 & -0.290 \\
99 & 13411.48313403 .98013403 .881 & -7.503 & -0.099 \\
100 & 13432.87213409 .06113409 .143 & -23.811 & 0.082 \\
\hline root-mean square residual & 4.47 & 0.340 \\
\hline maximum residual & 23.8 & 0.981 \\
\hline
\end{tabular}

The 100 lowest vibrational levels calculated with 146398 basis functions differ from those calculated with 73060 basis functions by less than $0.43 \mathrm{~cm}^{-1}$ on average. Most differences are less than $0.1 \mathrm{~cm}^{-1}$. The root-mean square residual is $0.34 \mathrm{~cm}^{-1}$, which confirms that our method works well. The memory cost of the largest calculation is 0.35 GB.

\section{CONCLUSION}

Collocation obviates the need for quadrature. Because the size of a direct product basis scales exponentially with the dimensionality of the problem, one requires a general procedure for choosing nondirect product bases, in order to make collocation useful for multidimensional problems. Some recipe for choosing collocation points, as many as there are basis functions, is also needed. To make the evaluation of matrix-vector products efficient, it is advantageous if both the basis and the point set have exploitable structure. This structure can be imposed by using Smolyak's ideas in conjunction with the general basis pruning condition,

$$
\mathcal{G}=\left\{\left.\left(n_{1}, \ldots, n_{D}\right)\right|^{(1)} G\left(n_{1}\right)+{ }^{(2)} G\left(n_{2}\right)+\ldots+{ }^{(D)} G\left(n_{D}\right) \leq b\right\}
$$


Iterative eigensolvers are more efficient and easier to use for a regular, rather than generalized, eigenvalue problem. The most straightforward way to use collocation with a regular matrix eigenvalue equation requires inverting $\mathbf{B}$, the matrix whose elements are basis functions evaluated at points. In this paper we show that by using so-called hierarchical basis functions, made from harmonic oscialltor basis functions and a Smolyak grid, it is easy and inexpensive to invert $\mathbf{B}$. The hierarchical functions are designed to make the $\mathbf{B}$ matrix for a single coordinate, ${ }^{(c)} \tilde{\mathbf{B}}$ (and therefore also ${ }^{(c)} \tilde{\mathbf{B}}^{-1}$ ) lower triangular. There is no need to store vectors with more elements than functions in the (pruned) basis. Results of calculations for $\mathrm{CH}_{2} \mathrm{NH}$ demonstrate that the method works.

\section{ACKNOWLEDGEMENTS}

The financial support of the Natural Sciences and Engineering Research Council is gratefully acknowledged. We thank Robert Wodraszka for proof reading.

\section{REFERENCES}

${ }^{1}$ W. Yang and A. C. Peet, Chem. Phys. Lett. 153, 98 (1988).

${ }^{2}$ A. C. Peet and W. Yang, J. Chem. Phys. 90, 1746 (1989).

${ }^{3}$ G. Avila and T. Carrington, J. Chem. Phys. 143, 214108 (2015).

${ }^{4}$ G. Avila and T. Carrington, J. Chem. Phys. 147, 064103 (2017).

${ }^{5}$ G. Avila and T. Carrington, J. Chem. Phys. 147, 144102 (2017).

${ }^{6}$ S. Manzhos and T. Carrington, J. Chem. Phys. 149, 204105 (2018).

${ }^{7}$ S. Manzhos, X. Wang, and T. Carrington, Chem. Phys. 509, 139 (2018).

${ }^{8}$ S. Manzhos and T. Carrington, J. Chem. Phys. 145, 224110 (2016).

${ }^{9}$ T. Carrington, J. Chem. Phys. 146, 120902 (2017).

${ }^{10}$ J. C. Light, I. P. Hamilton, and J. V. Lill, J. Chem. Phys. 82, 1400 (1985).

${ }^{11}$ J. C. Light and T. Carrington, in Adv. in Chem. Phys. (John Wiley \& Sons, Inc., 2007) pp. 263-310.

${ }^{12}$ G. Avila and T. Carrington, J. Chem. Phys. 137, 174108 (2012).

${ }^{13}$ G. Avila and T. Carrington, J. Chem. Phys. 134, 054126 (2011).

${ }^{14}$ G. Avila and T. Carrington, J. Chem. Phys. 131, 174103 (2009). 
${ }^{15}$ S. F. Boys, Proc. R. Soc. London, Ser. A 309, 195 (1969).

${ }^{16}$ J. Tennyson, Comp. Phys. Rep. 4, 1 (1986).

${ }^{17}$ B. T. Sutcliffe and J. Tennyson, Intern. J. Quantum Chem. 39, 183 (1991).

${ }^{18}$ M. J. Bramley and T. Carrington, J. Chem. Phys. 99, 8519 (1993).

${ }^{19}$ H.-G. Yu and J. T. Muckerman, J. Mol. Spectrosc. 214, 11 (2002).

${ }^{20}$ C. Fábri, E. Mátyus, and A. G. Császár, J. Chem. Phys. 134, 074105 (2011).

${ }^{21}$ A. G. Császár, C. Fábri, T. Szidarovszky, E. Mátyus, T. Furtenbacher, and G. Czakó, Phys. Chem. Chem. Phys. 14, 1085 (2012).

${ }^{22}$ C. Fábri, A. G. Császár, and G. Czakó, J. Phys. Chem. A 117, 6975 (2013).

${ }^{23}$ J. Sarka and A. G. Császár, J. Chem. Phys. 144, 154309 (2016).

${ }^{24}$ J. Sarka, A. G. Császár, and E. Mátyus, Phys. Chem. Chem. Phys. 19, 15335 (2017).

${ }^{25}$ T. Carrington, in Adv. Chem. Phys. (John Wiley \& Sons, Inc., 2018) pp. 217-243.

${ }^{26}$ U. Manthe and H. Köppel, J. Chem. Phys. 93, 345 (1990).

${ }^{27}$ R. Chen, G. Ma, and H. Guo, J. Chem. Phys. 114, 4763 (2001).

${ }^{28}$ R. J. Whitehead and N. C. Handy, J. Mol. Spectrosc. 59, 459 (1976).

${ }^{29}$ G. D. Carney, Adv. Chem. Phys. 37, 305 (1978).

${ }^{30}$ L. Halonen, D. W. Noid, and M. S. Child, J. Chem. Phys. 78, 2803 (1983).

${ }^{31}$ J. M. Bowman, S. Carter, and X. Huang, Int. Rev. Phys. Chem. 22, 533 (2003).

${ }^{32}$ X.-G. Wang and T. Carrington, J. Phys. Chem. A 105, 2575 (2001).

${ }^{33}$ R. Dawes and T. Carrington, Jr., J. Chem. Phys. 122, 134101 (2005).

${ }^{34}$ G. Avila and T. Carrington, J. Chem. Phys. 135, 064101 (2011).

${ }^{35}$ J. Cooper and T. Carrington, Jr., J. Chem. Phys. 130, 214110 (2009).

${ }^{36}$ H.-J. Bungartz and M. Griebel, Acta Numerica 13, 147 (2004).

${ }^{37}$ E. Novak and K. Ritter, Numerische Mathematik 75, 79 (1996).

${ }^{38}$ G. Wasilkowski and H. Wozniakowski, Journal of Complexity 11, 1 (1995).

${ }^{39}$ V. Barthelmann, E. Novak, and K. Ritter, Adv. Comp. Math. 12, 273 (2000).

${ }^{40}$ R. Wodraszka and T. Carrington, J. Chem. Phys. (submitted) (2019).

${ }^{41}$ M. Beck, A. Jäckle, G. A. Worth, and H.-D. Meyer, Phys. Rep. 324, 1 (2000).

${ }^{42}$ J. E. Dickens, W. M. Irvine, C. H. DeVries, and M. Ohishi, Astrophys. J. 479, 307 (1997).

${ }^{43}$ V. A. Basiuk, J. Phys. Chem. A 105, 4252 (2001).

${ }^{44}$ V. Vinogradoff, F. Duvernay, G. Danger, P. Theulé, and T. Chiavassa, Astro. \& Astrophysics 530, A128 (2011). 
${ }^{45}$ S. A. Smolyak, Sov. Math. Dokl. 4, 240 (1963).

${ }^{46}$ D. Lauvergnat and A. Nauts, Spectrochim. Acta, Part A 119, 18 (2014).

${ }^{47}$ G. Avila and T. Carrington, Chem. Phys. 482, 3 (2017).

${ }^{48}$ T. Halverson and B. Poirier, J. Phys. Chem. A 119, 12417 (2015).

${ }^{49}$ J. Brown and T. Carrington, J. Chem. Phys. 145, 144104 (2016).

${ }^{50}$ G. Avila, J. Oettershagen, and T. Carrington, Lecture Notes in Computational Science and Engineering , 1 (2018).

${ }^{51}$ F. Leja, Ann. Polon. Math. 4, 8 (1957).

${ }^{52}$ A. Narayan and J. D. Jakeman, SIAM Journal on Scientific Computing 36, A2952 (2014).

${ }^{53}$ P. Jantsch, C. G. Webster, and G. Zhang, IMA Journal of Numerical Analysis (2018).

${ }^{54}$ J. Valentin and D. Pflüger, in Lecture Notes in Computational Science and Engineering (Springer International Publishing, 2018) pp. 229-251.

${ }^{55}$ R. B. Lehoucq, D. C. Sorensen, and C. Yang, SIAM, Philadelphia, PA, 1998.

${ }^{56}$ T. Halverson and B. Poirier, J. Chem. Phys. 140, 204112 (2014).

${ }^{57}$ C. Pouchan and K. Zaki, J. Chem. Phys. 107, 342 (1997).

${ }^{58}$ J. M. Bowman, Chem. Phys. Lett. 180, 249 (1991). 